Article

\title{
We Need to Recreate Natural Philosophy
}

\author{
Nicholas Maxwell \\ Science and Technology Studies, University College London, Gower St., London WC1E 6BT, UK; \\ nicholas.maxwell@ucl.ac.uk
}

Received: 24 August 2018; Accepted: 25 September 2018; Published: 27 September 2018

\begin{abstract}
Modern science began as natural philosophy, an admixture of philosophy and science. It was then killed off by Newton, as a result of his claim to have derived his law of gravitation from the phenomena by induction. But this post-Newtonian conception of science, which holds that theories are accepted on the basis of evidence, is untenable, as the long-standing insolubility of the problem of induction indicates. Persistent acceptance of unified theories only in physics, when endless equally empirically successful disunified rivals are available, means that physics makes a persistent, problematic metaphysical assumption about the universe: that all disunified theories are false. This assumption, precisely because it is problematic, needs to be explicitly articulated within physics, so that it can be critically assessed and, we may hope, improved. The outcome is a new conception of science-aim-oriented empiricism - that puts science and philosophy together again, and amounts to a modern version of natural philosophy. Furthermore, aim-oriented empiricism leads to the solution to the problem of induction. Natural philosophy pursued within the methodological framework of aim-oriented empiricism is shown to meet standards of intellectual rigour that science without metaphysics cannot meet.
\end{abstract}

Keywords: natural philosophy; metaphysics; physics; problem of induction; physicalism; theoretical unity; philosophy of science; scientific method; scientific progress; pessimistic induction

\section{Newton Kills Natural Philosophy and Creates Modern Science}

Modern science began as natural philosophy, an admixture of philosophy and science. Today, we think of Galileo, Johannes Kepler, William Harvey, Robert Boyle, Christiaan Huygens, Robert Hooke, Edmond Halley, and of course Isaac Newton as trailblazing scientists, while we think of Francis Bacon, René Descartes, Thomas Hobbes, John Locke, Baruch Spinoza and Gottfried Leibniz as philosophers. That division is, however, something we impose on the past. It is profoundly anachronistic. At the time, they would all have thought of themselves as natural philosophers.

And then natural philosophy died. It split into science and philosophy. Both fragments suffered from the split, but philosophy suffered far more than science.

How and why did natural philosophy die? It was killed by Newton. Or rather, it was killed off by those Enlightenment and post-Enlightenment natural scientists who, following Newton, eschewed metaphysics, and sought to arrive at laws and theories solely on the basis of evidence.

Paradoxically, the first edition of Newton's Principia, published in 1687, was quite explicitly a great work of natural philosophy. There are, in the first edition, nine propositions all clearly labelled as "hypotheses," some quite clearly of a metaphysical character. By the third edition, the first two of these hypotheses become the first two "Rules of Reasoning," and the last five hypotheses, which concern the solar system, become the "Phenomena" of later editions. One hypothesis disappears altogether, and one other, not required for the main argument, is tucked away among theorems. In the third edition there are two further "rules of reasoning," both inductive in character. In connection with the second of these, Newton comments, "This rule we must follow, that the argument for induction may 
not be evaded by hypotheses" [1] (p. 400). Newton adds the following remarks concerning induction and hypotheses: "whatever is not deduced from the phenomena is to be called an hypothesis; and hypotheses, whether metaphysical or physical ... have no place in experimental philosophy. In this philosophy, particular propositions are inferred from the phenomena, and afterwards rendered general by induction. Thus it was that ... the laws of motion and of gravitation were discovered" [1] (p. 547). In these and other ways, Newton sought to transform his great work in natural philosophy into a work of inductive science.

Newton hated controversy. He knew his law of gravitation was profoundly controversial, so he doctored subsequent editions of his Principia to hide the hypothetical, metaphysical and natural philosophy elements of the work, and make it seem that the law of gravitation had been derived, entirely uncontroversially, from the phenomena by induction. Because of Newton's immense prestige, especially after his work was taken up by the French Enlightenment, subsequent natural philosophers took it for granted that success required they proceed in accordance with Newton's methodology. Laws and theories had to be arrived at, or at least established, by means of induction from phenomena. Metaphysics and philosophy had become irrelevant, and could be ignored. Thus was modern science born, and natural philosophy, which had given rise to modern science in the first place, was quietly forgotten. ${ }^{1}$

Newton's inductivist methodology is still with us. It is known today as "inference to the best explanation" [3]. (Newton did not ignore explanation. His Rules of Reasoning stressed that induction required one to accept the theory that is simplest and, in effect, gives the best explanation of phenomena.) Scientists today may not hold that theories can be "deduced" from phenomena by induction, but they do hold that evidence alone (plus explanatory considerations) decides what theories are accepted and rejected in science. They take for granted a doctrine that may be called standard empiricism: evidence decides in science what theories are to be accepted and rejected, with the simplicity, unity or explanatory power of theories playing a role as well, but not in such a way that the world, or the phenomena, are assumed to be simple, unified or comprehensible. The crucial point, inherited from Newton, is that no thesis about the world can be accepted as a part of scientific knowledge independently of evidence, let alone in violation of evidence. In essence, Newton's methodology of evidence and theory still dominates the scene. The split between science and philosophy, which was one outcome, persists.

But Newton bequeathed to philosophy a fundamental problem about the nature of science that, for most philosophers, remains unsolved today. It is the problem of induction, brilliantly articulated by David Hume. ${ }^{2}$ This problem cannot be solved as long as we hold on to Newton's conception of science. Hume, in effect, refuted the Newtonian conception of science-the standard empiricist conception scientists and non-scientists still take for granted today. In order to solve Hume's problem of induction, we must reject this orthodox conception of science we have inherited from Newton, and adopt natural philosophy instead, a conception of science that brings science and metaphysics ${ }^{3}$ intimately together again. We need to bring about a revolution in science, and in our whole conception of science - one that leads to the recreation of natural philosophy—if we are to have a kind of science (or natural philosophy, rather) that is free of Hume's problem of induction. Science may seem to be more intellectually rigorous than natural philosophy because it dissociates itself from questionable metaphysical hypotheses, whereas natural philosophy does not. Actually, it is all the other way around. Because science dissociates itself from metaphysics, it is unable to provide a solution to the problem of induction. It lacks intellectual rigour. Natural philosophy, on the other hand, because it openly

1 For a much more detailed account of Newton's involvement in the demise of natural philosophy and the rise of science, see [2] (Chapters 1 and 2).

2 See [4] (Volume 1, Part III, especially Section VI). For a good, fairly recent discussion of the problem, see [5].

3 As I employ the term, a thesis is "metaphysical" if it is about the nature of the universe but is too imprecise to be empirically verifiable or falsifiable. 
acknowledges metaphysical hypotheses, can solve the problem of induction. It is more rigorous than science. Reason demands that we push through a revolution in our whole understanding of the nature of science.

\section{Natural Philosophy Required to Solve Hume's Problem of Induction}

The problem of induction can be put like this: How can evidence ever verify a scientific theory? In particular, how can any theory of physics ever be verified by evidence? Given any such theory, however "well established" it may be by evidence, there will always be endless rival theories that will fit the available evidence even better. On what grounds can all these rival theories be ignored? Granted that they always exist, how can any physical theory ever be empirically confirmed by evidence, to any degree of probability above zero? How can theories even be selected by evidence, let alone verified?

Proponents of "induction to the best explanation" may think they have an answer. Physics only ever accepts theories that are explanatory or unified. All the rivals that can readily be formulated that fit the phenomena even better than any accepted physical theory are all hopelessly, grotesquely ad hoc, disunified and non-explanatory, and are ignored for that reason. If, for the sake of argument, we take Newton's law of gravitation as the accepted theory, then one kind of grotesquely ad hoc, disunified rival theory would postulate, arbitrarily, that at some time in the future, Newton's law will become an inverse cube law; another kind of theory would postulate that, for systems never observed (for example, gold spheres with a mass of 10,000 tons), gravitation becomes a repulsive force. Independent postulates that have been empirically confirmed independently are added on to these grotesquely disunified rivals to make them fit phenomena (for the time being) even more successfully than Newton's law. All such laws and theories, grotesquely disunified versions of Newton's theory that fit the evidence even better than Newton's theory, are ignored by physics in practice precisely because these rivals are all hopelessly disunified.

Does this solve the problem-Hume's problem of induction? It does not. We may grant that a theory of physics, in order to be accepted, must satisfy two requirements. It must be (i) sufficiently empirically successful, and it must be (ii) sufficiently unified-the second requirement even over-riding the first (in that unified theories are persistently preferred to empirically more successful but disunified rivals). But at once two new problems demand attention. First, what does it mean to declare of a theory that it is unified (or disunified)? Second, what possible justification can there be for persistently accepting unified theories when empirically more successful disunified rivals can always be formulated?

Philosophers of science have long struggled to solve these two problems, especially the second one. ${ }^{4}$ But these attempts have almost always missed the following crucial, extremely simple point. If physics only ever accepts unified theories when endlessly many empirically more successful disunified rivals are available, then physics must thereby make a big assumption about the nature of the universe: the universe is such that all disunified theories, whatever their empirical success may be, are false. There is some kind of underlying unity in nature. ${ }^{5}$

Some philosophers have indeed tried to solve the problem of induction by proposing that science does indeed adopt some sort of metaphysical conjecture concerning the uniformity or unity of natural phenomena: see [17] (pp. 255-293); [18]; [19] (pp. 254-268); [20] (pp. 100-101). Most philosophers have reacted to these suggestions with scorn. Thus Bas van Fraassen has remarked, "From Gravesande's axiom of the uniformity of nature in 1717 to Russell's postulates of human knowledge in 1948, this has been a mug's game" [21] (pp. 259-260).

In fact it is all the other way around. Precisely because this assumption of underlying unity is a pure conjecture, which may well be false, it is absolutely essential that it is acknowledged by physics so

4 For the solution to the first problem - the problem of what it means to say of a physical theory that it is unified-see [6] (Chapters 3 and 4); [7] (Appendix, Section 2); or [8] (Chapter 5).

5 This simple but, it seems to me, decisive point, with profound ramifications for our understanding of the nature of science, is one that I have sought to communicate for decades: see [2,6-16]. 
that it can be critically assessed and, we may hope, improved. It is a mug's game not to acknowledge this profoundly problematic metaphysical presupposition implicit in physicists' persistence acceptance of unified theories only, when many empirically more successful disunified rivals exist. Intellectual rigour and rationality demand that this problematic implicit assumption be made explicit so that it can be critically assessed and, we may hope, improved.

Most philosophers of science take it for granted that appealing to any such metaphysical thesis concerning uniformity or unity "is a mug's game" because there can be no hope of demonstrating that any such thesis is true, or even probably true. It could only be an unfounded conjecture. But they thereby profoundly miss the point. To repeat: it is precisely because this metaphysical thesis, this presupposition, is only an unfounded conjecture, and a profoundly problematic one at that, that it is so vital that it be made explicit within physics so that it can be subjected to sustained critical scrutiny in an attempt to improve it. It is vital to make the assumption explicit because, though almost bound to be false given the specific version accepted by physics at any stage in its development, it nevertheless exercises a profound influence over theoretical physics-over what kind of new theories physicists seek to develop, and over what theories they accept.

Three points need to be appreciated: (1) The metaphysical conjecture of uniformity or unity is actually made by physics, whether this is recognized or not. It is made as a consequence of the way physics only ever accepts unified theories when many empirically more successful disunified rivals exist. (2) The conjecture is, at best, an unfounded conjecture, quite likely to be false. Given the specific version of the conjecture implicitly accepted by physics at any stage in its development, it is almost bound to be false-as the historical record reveals. ${ }^{6}$ (3) The conjecture concerning uniformity or unity, though quite likely to be false, nevertheless exercises an immense influence over both the search for new theories, and what theories are accepted and rejected. It is this combination of (1) being implicitly accepted by physics, (2) being no more than an unfounded conjecture quite likely to be false, and (3) nevertheless exercising an immense influence over physics, which makes it so vital, for physics itself, that the conjecture is made explicit so that it can be explicitly criticized and, we may hope, improved.

The upshot is that we need to put physics and metaphysics together again to create a modern version of natural philosophy. We need to recreate the vision of science of Galileo, Kepler, Boyle, Huygens, and above all of Newton of the first edition of the Principia, a vision that sees science wedded to problematic metaphysical speculations about the ultimate nature of the physical universe. These speculations need to be subjected to sustained imaginative and critical exploration, as an integral part of the scientific enterprise, in an attempt to improve a thesis that can be accepted at any stage in the development of physics.

But how, it may be asked, can the problematic metaphysical presuppositions of physics be improved? What methods can best facilitate such improvement?

Elsewhere, I have shown in detail how this problem is to be solved. We need to adopt, and put into scientific practice, a view that I have called aim-oriented empiricism (Figure 1): see [2,6-16].

6 See for example [8] (pp. 64-65). 


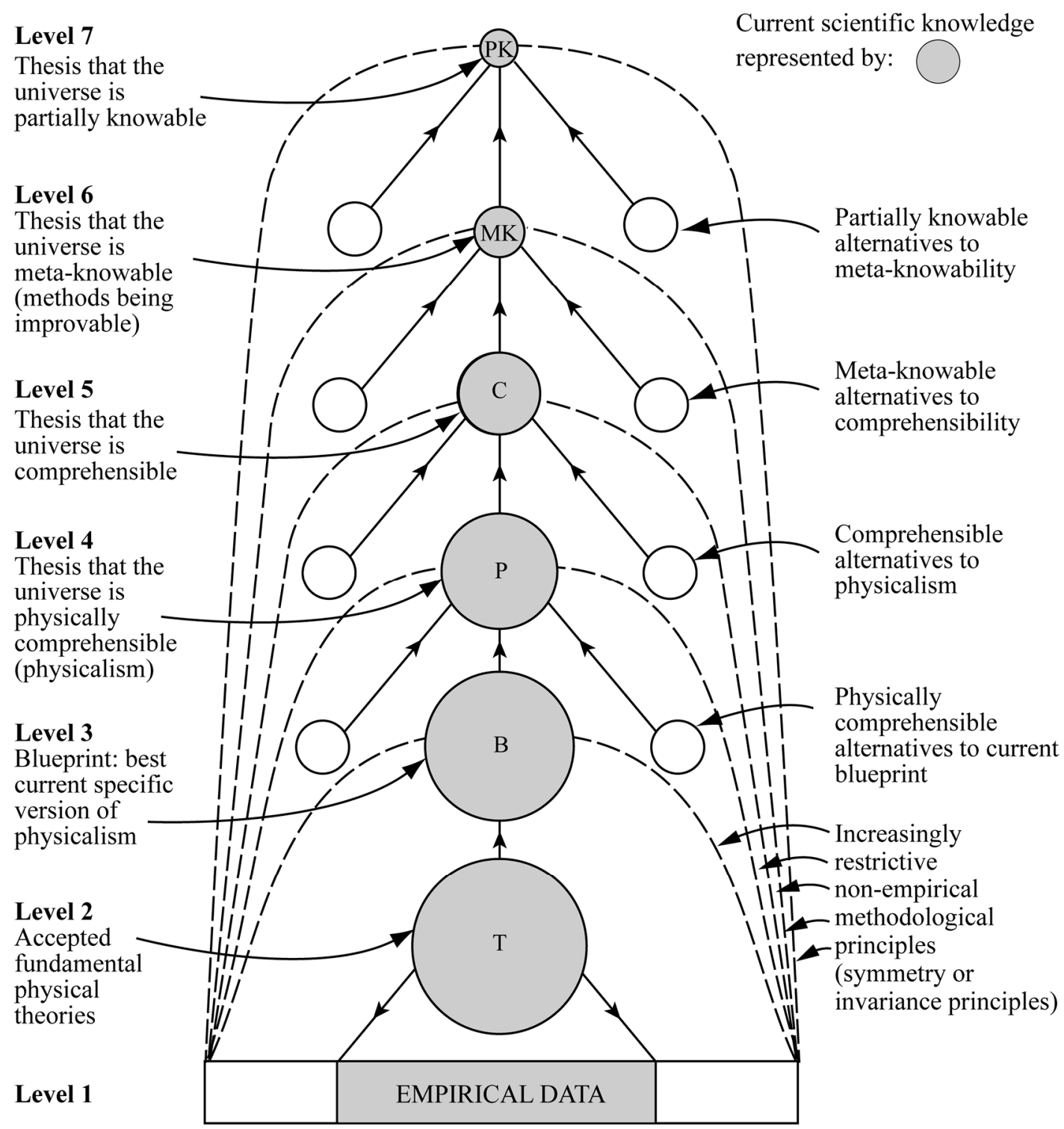

Figure 1. Aim-oriented empiricism.

\section{Aim-Oriented Empiricism: The Methodological Backbone of the New Natural Philosophy}

The basic idea of aim-oriented empiricism (AOE) is that we need to represent the influential, problematic metaphysical presupposition of physics concerning underlying unity in the form of a hierarchy of assumptions. As we go up this hierarchy, assumptions become less and less substantial, and so more and more likely to be true, and more and more nearly such that their truth is required for science, or the pursuit of knowledge to be possible at all. In this way, we create a framework of assumptions (and associated methods) high up in the hierarchy, very likely to be true, within which much more substantial assumptions (and associated methods) low down in the hierarchy, very likely to be false, can be critically assessed and, we may hope, improved.

How does this hierarchical framework facilitate improvement of metaphysical presuppositions of physics, so that they become closer to the truth, more fruitful for physics itself? It does so by concentrating imaginative exploration and critical scrutiny where it is most likely to be fruitful for scientific progress, low down in the hierarchy of assumptions where assumptions are most likely to be false. It does so by ensuring that new possible assumptions, worth considering, low down in the hierarchy, are fruitfully constrained, partly by assumptions higher up in the hierarchy, partly by physical theories that have met with the greatest empirical success. Those metaphysical 
assumptions, low down in the hierarchy, are chosen that stimulate, or are associated with, the most empirically progressive research programmes in physics, or hold out the greatest hope of that. In these ways, the hierarchical framework of AOE facilitates improvement in metaphysical theses that are accepted low down in the hierarchy. As theoretical knowledge in physics improves, metaphysical presuppositions improve, and even lead the way. There is something like positive feedback between improving metaphysical assumptions and associated methods, and improving theoretical knowledge in physics. As we improve our scientific knowledge and understanding about the universe, we correspondingly improve the nature of science itself. We improve methods for the improvement of knowledge. ${ }^{7}$

AOE is depicted in the Figure. ${ }^{8}$ At the top, at level 7, there is the thesis that the universe is such that we can acquire some knowledge of our local circumstances sufficient to make life possible. If this is false, we have had it, whatever we assume. It can never, in any circumstances, imperil the pursuit of knowledge to accept this thesis as an item of scientific knowledge-and may help promote scientific knowledge- - even though we have no reason to hold it to be true, or probably true. Thus, we are rationally entitled to accept the thesis as a part of scientific knowledge even though the thesis is a pure conjecture. We are rationally entitled to accept the thesis on pragmatic grounds: in accepting the thesis, we have nothing to lose, and may have much to gain.

At level 6 there is the thesis that the universe is such that we can learn how to learn. It is not just that we can acquire new knowledge. We can acquire new knowledge about how to acquire new knowledge. The universe is such that we can make a discovery about it which makes it possible for us to improve our methods for the improvement of knowledge. This thesis deserves to be accepted, again, on pragmatic grounds: we have little to lose, and may have much to gain, in our search for improved knowledge about the universe.

One possibility, which accords with the level 6 thesis of meta-knowability, is that the universe is comprehensible in some way. The universe is such that there is some standard kind of explanation as to why natural phenomena occur as they do. It might be that natural phenomena occur in response to the intentions of gods; or of one God; or in accordance with some cosmic purpose. Or it might be that they occur in compliance with some cosmic "computer programme" as some have suggested. Or it might be that natural phenomena occur as they do to accord with some unified pattern of physical law. ${ }^{9}$

This level 5 thesis of comprehensibility accords with the level 6 thesis of meta-knowability because, if the level 5 thesis is true, then there is every hope that, by choosing and developing that version of the comprehensibility thesis that best stimulates progress in empirical knowledge, it will be possible progressively to improve methods for the improvement of knowledge, as we proceed. Granted we have accepted the level 6 thesis, it makes good sense to accept the level 5 thesis, provisionally at least, until all more specific versions of the thesis provide no help whatsoever with improving empirical knowledge. But if, on the contrary, some specific version of the level 5 thesis of comprehensibility seems to facilitate rapid improvement in empirical knowledge, then this deserves to be accepted, in accordance with theses at levels 6 and 5, until something better turns up.

At level 4 there is the thesis that the universe is physically comprehensible. The universe is such that the as-yet undiscovered true physical theory of everything is unified. A physical theory is unified if its content, what it asserts about the world, is the same throughout all the actual and possible physical

7 This positive feedback process of improving presuppositions and methods, or aims and methods, in the light of what stimulates empirical progress and what does not, has actually gone on in physics, and in natural science more generally-or we would still be stuck with Aristotelian science. But because the scientific community has taken standard empiricism for granted, it has only been possible for this scientifically fruitful, positive feedback process to proceed in a somewhat furtive, constrained manner.

8 In what follows I give only a brief sketch of AOE, and reasons for accepting AOE. The best detailed argument for AOE is given in [8]. See also [2] (especially Chapter 5); and [22]. I must stress, however, that AOE was first expounded and argued for in publications that appeared much earlier: see [6,9-13].

9 This last possibility can be interpreted in the way that has been argued for in [23]. 
systems to which it applies. If these physical systems divide up into $\mathrm{N}$ groups such that, the content of the theory is the same throughout any one group, but different from the content in all the other groups, then the theory is disunified to degree $\mathrm{N}$. For unity we require $\mathrm{N}=1$. Above we have seen that a theory can be disunified in different ways: its content (1) can differ at different times, or space-time regions; or its content (2) can differ from one kind of physical system to the next. There are further ways in which the content of a physical theory can change as we move from one range of possible phenomena to which the theory applies to another range. (3) The theory may postulate one or more spatially restricted objects, each with its own unique dynamic properties. (4) The theory may postulate two or more forces, one force law operating in one range of possible phenomena, another force law operating in another range. (5) The theory may postulate one force, but two or more kinds of physical entity, with different masses or charge, for example, one kind of entity in one range of possible phenomena, another kind of entity in another range of phenomena. (6) A theory disunified in ways (4) or (5) may be unified if the theory satisfies a symmetry that is such that a symmetry transformation has the effect of transforming strengths of forces from one kind to another, or the effect of transforming one kind of physical entity into another kind. Elsewhere I have distinguished eight different ways in which a physical theory can be disunified, all variants of the same basic idea of theory disunity or unity: see [6] (Chapters 3 and 4); [7] (Appendix, Section 2); [8] (Chapter 5). The level 4 thesis that the universe is physically comprehensible is to be interpreted as asserting that the true physical theory of everything is unified, with $\mathrm{N}=1$, in the strongest of the eight ways in which a theory can be unified.

Granted that theses at levels 6 and 5 have been accepted, there are overwhelming grounds for accepting this level 4 thesis of physical comprehensibility, or physicalism. ${ }^{10}$ For the theses at levels 6 and 5 imply that if a precise version of the level 5 thesis begins to stimulate the growth of empirical knowledge, then that thesis deserves to be accepted and pursued-until something better turns up. It is worth reminding ourselves, at this point, just how astonishingly empirically fruitful physicalism has been over the centuries. As physics has evolved since the time of Kepler and Galileo, the totality of accepted fundamental physical theory has become (a) vastly more extensive in predictive scope, and at the same time has brought about astonishing theoretical unification in the dramatically increasing range of phenomena known to us.

A metaphysical thesis such as physicalism is not empirically verifiable or falsifiable. It may, however, be empirically fruitful. A metaphysical thesis, $\mathrm{M}$, is empirically fruitful if there is a succession of physical theories, $T_{1}, \ldots T_{n}$, that are increasingly successful empirically (successfully predicting ever wider ranges of phenomena with ever increasing accuracy), the succession of theories being such that they draw ever closer to capturing $\mathrm{M}$ as a testable physical theory. The whole way in which theoretical physics has developed since Kepler and Galileo renders physicalism astonishingly empirically fruitful. For all advances in theory in physics since the scientific revolution have been advances in unification.

Thus Newtonian theory (NT) unifies Galileo's laws of terrestrial motion and Kepler's laws of planetary motion (and much else besides). Maxwellian classical electrodynamics, (CEM), unifies electricity, magnetism and light (plus radio, infrared, ultra violet, $\mathrm{X}$ and gamma rays). Special relativity (SR) brings greater unity to CEM, in revealing that the way one divides up the electromagnetic field into the electric and magnetic fields depends on one's reference frame. SR is also a step towards unifying NT and CEM in that it transforms space and time so as to make CEM satisfy a basic principle fundamental to NT, namely the (restricted) principle of relativity. SR also brings about a unification of matter and energy, via the most famous equation of modern physics, $\mathrm{E}=\mathrm{mc}^{2}$, and partially unifies space and time into Minkowskian space-time. General relativity (GR) unifies space-time and gravitation, in that, according to GR, gravitation is no more than an effect of the curvature of space-time. Quantum theory $(\mathrm{QM})$ and atomic theory unify a mass of phenomena

10 "Physicalism" has been interpreted in a number of ways by various philosophers of science. Here it means simply: the universe is such that the true physical theory of everything is unified in the strongest sense with $N=1$, see [8] (Chapter 5). 
having to do with the structure and properties of matter, and the way matter interacts with light. Quantum electrodynamics unifies QM, CEM and SR. Quantum electroweak theory unifies (partially) electromagnetism and the weak force. Quantum chromodynamics brings unity to hadron physics (via quarks) and brings unity to the eight kinds of gluons of the strong force. The standard model (SM) unifies to a considerable extent all known phenomena associated with fundamental particles and the forces between them (apart from gravitation). The theory unifies to some extent its two component quantum field theories in that both are locally gauge invariant. ${ }^{11}$ Current research programmes in fundamental theoretical physics seek to unify SM and GR. ${ }^{12}$

In short, all advances in fundamental theory since Galileo have invariably brought greater unity to theoretical physics in one or other, or all, of the different kinds of unity I have distinguished, from 1 to 8 . All successive theories have increasingly successfully exemplified and given precision to physicalism (as interpreted here) to an extent that cannot be said of any rival metaphysical thesis, at that level of generality. The whole way theoretical physics has developed points towards physicalism, in other words, as the goal towards which physics has developed. ${ }^{13}$

Granted acceptance of theses at levels 6 and 5, and granted the way theoretical physics has developed since Galileo, grounds for accepting physicalism at level 4 become irresistible.

At level 3 that metaphysical thesis is accepted that is as specific a version of physicalism as possible that (a) accords with physicalism, and (b) is the best current conjecture as to how accepted physical theories at level 2 are to be unified. What ought to be accepted at level 3 today constitutes a vital, open problem for theoretical physics and the metaphysics of physics. One possibility is string theory, or M-theory. Another is what I have called Lagrangianism. ${ }^{14}$

At level 2 those physical theories are accepted (a) that meet with sufficient empirical success, and (b) that sufficiently enhance the type and degree of unity of the totality of fundamental physical theory, and thus accord sufficiently well with the level 4 thesis of physicalism. At level 1 we have accepted empirical data-what are judged to be repeatable effects, and thus low-level empirical laws.

There are, as I have sought to indicate, very strong arguments for AOE, and very strong arguments against its rivals, all versions of the orthodox view of standard empiricism (SE). SE acknowledges that persistent preference is given in physics to unified theories, but dishonestly fails to acknowledge that that means physics makes a persistent assumption about the universe: it is such that disunified theories are false. SE fails to provide an acceptable account of what the unity of a physical theory is, ${ }^{15}$ and fails to justify persistent acceptance of unified theories, especially given that many empirically more successful disunified rivals are always available. SE fails to solve the problem of induction.

By contrast, AOE acknowledges that physics does make a big, highly problematic, influential metaphysical assumption about the universe. The hierarchical structure of assumptions and associated methods of $\mathrm{AOE}$ is designed to facilitate development and acceptance of assumptions, low down in the hierarchy, most likely to promote scientific progress of theoretical physics, progress in knowledge and understanding. Not only does AOE make thoroughly explicit the considerations that govern acceptance of theories in physics (something SE cannot do). In addition, it provides a rational, if fallible, method for the discovery of new physical theories. ${ }^{16}$ AOE specifies precisely what it is for a theory to be unified, and justifies acceptance of theories unified in this sense-granted they are sufficiently empirically successful. The hierarchical framework of AOE makes it possible for physics to modify its

11 See [10] (2007, pp. 420-421).

12 For clarification of details and further discussion, see [6] (pp. 80-89, 131-140, and 257-265, and additional works referred to therein). See also [7] (Appendix, Section 2); [8] (Chapter 5).

13 See [10] (2007, p. 421).

14 See [6] (pp. 98-90); or [8] (pp. 127-128, Note 14).

15 Given the account of theory unity indicated here, it is dazzlingly clear that persistent acceptance of unified theories in this sense must inevitably commit physics to making a big metaphysical assumption about the world (the world is such that disunified theories are false whatever their empirical success may be). SE cannot very well acknowledge this account of theory unity for, to do so, destroys SE.

16 See [6] (pp. 219-223); [11] (pp. 275-305); and above all [2] (Chapter 5). 
metaphysical assumptions and associated methods in the light of what it learns about the nature of the universe. As our knowledge improves, our knowledge about how to improve knowledge improves as well, a positive feedback feature of AOE that helps explain the explosive growth of scientific knowledge. And, in addition to all this, AOE solves the problem of induction: [6] (Chapter 5); [11] (pp. 61-79); and especially [8] (Chapter 9). There are good reasons why metaphysical theses at the various levels of AOE deserve to be accepted-briefly indicated above.

AOE ought to be adopted and implemented by scientists and philosophers of science alike. I hope the case for AOE, sketched here, will at least arouse interest. A convincing case for AOE is spelled out in some detail in [8], with [2] giving an account of some of the implications of putting AOE into scientific practice. ${ }^{17}$

There is, however, a well-known and apparently devastating objection to AOE-to the claim, in particular, that $\mathrm{AOE}$ solves the problem of induction. According to AOE, those metaphysical theses (low down in the hierarchy of theses) are accepted that best accord with accepted physical theories; at the same time, those physical theories are accepted that best accord with the metaphysical theses. Acceptance of empirically successful physical theories is justified by an appeal to metaphysical theses; acceptance of these metaphysical theses is then justified by an appeal to the astonishing success of physics! But such an argument is, it seems, viciously circular. It presupposes just that which it sets out to justify. What makes matters worse, is that AOE has this circularity built into it quite explicitly; it is even upheld as its greatest virtue and triumph. The whole point of the view, after all, is to facilitate the critical assessment of theses low down in the hierarchy in the light of the empirical success and failure of science. How can AOE survive this devastating criticism of vicious circularity?

The solution to this problem stems from the level 6 metaphysical thesis of meta-knowability. Permitting metaphysical assumptions to influence what theories are accepted, and at the same time permitting the empirical success of theories to influence what metaphysical assumptions are accepted, may (if carried out properly), in certain sorts of universe, lead to genuine progress in knowledge. Meta-knowability is to be interpreted as asserting that this is just such a universe. And furthermore, crucially, reasons for accepting meta-knowability make no appeal whatsoever to the success of science. In this way, meta-knowability legitimizes the potentially invalid circularity of AOE. ${ }^{18}$

In what follows, we need to consider possible universes in which the top two theses in the hierarchy of theses of AOE are true, but everything below these may be false. Something like the meta-methodology of AOE can meet with success, so that we can improve, not just knowledge, but also knowledge about how to improve knowledge, without being restricted to universes in which the level 4 thesis of physicalism is true, or even the level 5 thesis of comprehensibility. We need a generalized version of AOE-generalized AOE or GAOE-which has the hierarchical structure AOE, agrees with $\mathrm{AOE}$ as far as theses at levels 6 and 7 are concerned, but is open about what theses obtain at levels 2 to 5 (or even 1 to 5 ).

Relative to an existing body of knowledge and methods for the acquisition of new knowledge, possible universes can be divided up, roughly, into three categories: (i) those that are such that the meta-methodology of AOE, or GAOE, can meet with no real success, in the sense that new metaphysical ideas and associated methods for the improvement of knowledge cannot be put into practice so that success is achieved; (ii) those that are such that AOE, or GAOE, appears to be successful for a time, but this success is illusory, this being impossible to discover during the period of illusory success; and (iii) those that are such that GAOE, and even AOE, can meet with genuine success. Meta-knowability asserts that our universe is a type (i) or (iii) universe; it rules out universes of type (ii).

Meta-knowability asserts, in short, that the universe is such that AOE, or GAOE, can meet with success and will not lead us astray in a way in which we cannot hope to discover by normal methods of

17 For earlier expositions of the argument for AOE see [6,7,9,11-16]; [10] (pp. 94-100 and Chapter 9).

18 See [10] (2007, p. 414). 
scientific inquiry (as would be the case in a type (ii) universe). If we have good grounds for accepting meta-knowability as a part of scientific knowledge-grounds that do not appeal to the success of science-then we have good grounds for adopting and implementing AOE, or GAOE, (from levels 5 to 2). Meta-knowability, if true, does not guarantee that AOE will be successful. Instead it guarantees that AOE will not meet with illusory success, the illusory character of this apparent success being such that it could not have been discovered by any means whatsoever before some date is reached. ${ }^{19}$

We do, however, have good reasons for accepting meta-knowability that make no appeal to the success of science; meta-knowability renders the ostensibly invalid circularity of AOE valid and justified.

As I have already indicated, others have sought to solve the problem of induction by arguing for acceptance of metaphysical theses concerning the uniformity or unity of nature on what may be called "pragmatic" grounds: it is in the interests of the pursuit of knowledge of factual truth to accept the thesis in question, even though we have no reason to hold that the thesis is true, or probably true. ${ }^{20}$ These attempts all appeal to just one metaphysical thesis, on one level. As a result, they can only provide one kind of reason for the acceptance of the thesis in question. The great advantage of AOE is that the metaphysical assumptions of physics are on five different levels. This means different sorts of reason can be given for accepting metaphysical theses at different levels. The top two theses are accepted for pragmatic reasons: it is in the interests of the pursuit of truth to accept these theses. The bottom three metaphysical theses are accepted because of their potential or actual empirical fruitfulness.

Three reasons can be given as to why AOE is absolutely essential for the solution to the problem of induction:

1. In order to solve the problem, we need an intellectually rigorous conception of science. AOE alone has the required rigor, in that it alone acknowledges and provides the means to improve problematic metaphysical assumptions of science.

2. The hierarchical structure of AOE is an essential requirement for the solution to the problem. It is needed, because quite different reasons need to be given for accepting theses, at the five different levels of the view. If these different levels are collapsed into one level, this can no longer be done.

3. AOE is required to solve the apparent vicious circularity involved in justifying acceptance of physical theory by an appeal to metaphysics, and then justifying acceptance of this same metaphysics by an appeal to the empirical success of physical theory. AOE alone solves this problem by accepting, at level six, a metaphysical thesis that asserts, in effect, that the universe is such that this apparently viciously circular procedure can meet with success, acceptance of this metaphysical thesis being justified in a way that makes no appeal to the empirical success of science whatsoever.

\section{Revolutionary Implications of AOE for Science and the Philosophy of Science}

In my publications I have demonstrated in some detail that the above considerations in support of $\mathrm{AOE}$, have the following substantial implications:

1. AOE needs to be put into scientific practice in order to strengthen the intellectual integrity and success of science. The outcome would be a new kind of science, more rigorous and of greater intellectual and humanitarian value. Science itself would change, and be improved. ${ }^{21}$

2. All versions of standard empiricism are untenable..$^{22}$

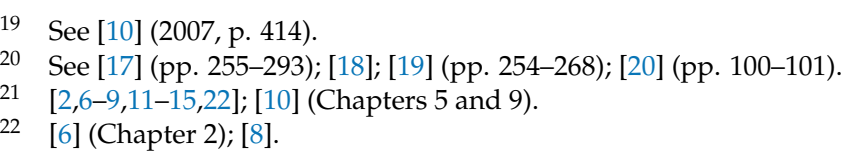


3. The relationship between science and the philosophy of science would be transformed. Philosophy of science would become an integral part of science itself. ${ }^{23}$

4. AOE reveals that science has already established, as a part of theoretical knowledge, the metaphysical thesis physicalism (as I have called it, the level 4 thesis of the diagram). ${ }^{24}$ This asserts that the universe is physically comprehensible-that is, it is such that there is a yet-to-be-discovered physical "theory of everything" that is unified and true.

5. Physicalism, though incompatible with current knowledge in physics at the level of theory (general relativity plus the standard model), is nevertheless one of the most secure items of theoretical knowledge in physics that we have, so secure that any theory that clashes too severely with it is rejected, whatever its empirical success may be. ${ }^{25}$

6. Scientific method is revealed to have a hierarchical structure corresponding to the hierarchical structure of metaphysical presuppositions, or aims, of science. It is this hierarchical structure that makes it possible for methods, high up in the structure, to control evolving methods, low down in the structure. ${ }^{26}$

7. AOE carries the implication that orthodox quantum theory, or indeed any version of quantum theory that is about the result of measurement only and not, in the first instance, about quantum systems as such, is seriously defective (it lacks unity). ${ }^{27} \mathrm{~A}$ fully micro-realistic version of quantum theory, probabilistic or deterministic, needs to be developed. ${ }^{28}$

8. The so-called "pessimistic induction" is no grounds for pessimism at all. The way in which physics has proceeded, from Newton to today (even though from one false theory to another), is just the way physics would proceed were it to be making splendid progress (and AOE is correct). ${ }^{29}$

9. AOE facilitates the progressive improvement of the metaphysics of science in the light of (a) a priori, and quasi a priori considerations (e.g., having to do with unity), and (b) considerations that have to do with empirical fruitfulness-the extent to which the metaphysical thesis in question has led to an empirically progressive scientific research programme. ${ }^{30}$ According to AOE, science improves its metaphysical assumptions and associated methods as it improves its knowledge: there is something like positive feedback between them (which helps account for the explosive growth in scientific knowledge). The metaphysics of physics becomes an integral part of physics itself.

10. AOE solves the problem of induction - and is required to solve the problem. ${ }^{31}$

11. The problem of what it means to say of a physical theory that it is unified is solved within the framework of AOE. This solution provides the means to partially order physical theories with respect to unity. Unity and simplicity are sharply distinguished. ${ }^{32}$

12. Eight kinds of theoretical unity are distinguished, increasingly demanding versions of the basic notion of theory unity. ${ }^{33}$

13. Unification in theoretical physics is of two kinds: unification by (a) annihilation, and by (b) synthesis. ${ }^{34}$

\footnotetext{
[7] (pp. 34-51); [10] (pp. 231-232, 235 and 240-42); [12].

[6] (especially pp. 19-20, 26, 98 and Chapter 5); [8] (Chapter 9).

See Note 24.

[6] (pp. 29-30); [7] (pp. 42-47); [8] (Chapter 10); [9]; [11] (pp. 275-305).

[6] (pp. 231-232); [24] (pp. 276-278); [25] (pp. 2-3).

28 For my own efforts at developing a fully micro-realistic, fundamentally probabilistic version of quantum theory, empirically testable, and free of the defects of orthodox quantum theory, see [24-30].

[6] (pp. 211-212); [8] (pp. 83-86).

For a lucid exposition of this point see [8] (pp. 74-82). See also [2] (Chapter 5).

31 For my early attempts at solving the problem of induction, see Maxwell [6] (Chapter 5); [9]; [10] (pp. 218-230); [11] (pp. 61-79). For a much improved exposition of the solution provided by AOE, see [8] (Chapter 9).

32 [6] (Chapters 3 and 4); [7] (Appendix, Section 2); and [8] (Chapter 5).

33 See Note 32.

34 [6] (pp. 125-126).
} 
14. AOE solves the problem of why physics is justified in preferring unified theories to disunified ones. ${ }^{35}$

15. The problem of verisimilitude is solved within the framework of AOE. ${ }^{36}$

16. AOE provides physics-and science more generally-with a fallible, non-mechanical (i.e., non-algorithmic) but rational method for the discovery of good new theories. ${ }^{37}$

17. AOE is a synthesis of, and a great improvement over, the views of Popper, Kuhn and Lakatos. ${ }^{38}$

18. Instrumentalism (or constructive empiricism) is untenable because it cannot do justice to the requirement of unity. Unity demands scientific realism. ${ }^{39}$

19. AOE clarifies the role of symmetry principles in theoretical physics and provides a justification for the role that they play: a symmetry of a theory is a facet of its unity. The requirement that a physical theory, in order to be acceptable, must satisfy symmetry principles stems from (is an aspect of) the requirement that it be unified. ${ }^{40}$

20. AOE does justice to the fact that different branches of natural science employ different methods; it does justice to the fact that methods of a particular science evolve as that science makes progress over time: at the same time, AOE specifies meta-methods that are, ideally, common to all branches of science at all times, and as a result justifies adoption of differing and evolving methods. ${ }^{41}$

The upshot of all this is that a revolution is required in both science and philosophy. Scientists and philosophers need to collaborate in transforming research and teaching in both science and philosophy, so that the two branches of inquiry are brought together to create a modern version of natural philosophy.

\section{Broader Considerations}

I have argued so far that the long-standing failure of philosophy to solve one of its fundamental problems-the problem of induction - has led to the persistence of the split between science and philosophy, the failure to put science, metaphysics and philosophy back together again to create a modern version of natural philosophy. Another long-standing failure of philosophy has had even broader repercussions. This time, the failure is not to solve one of the fundamental problems of philosophy. It is the much more elementary failure to formulate the problem properly.

The problem I have in mind ought to be formulated like this: How can our human world, the world as it appears to us, the world we live in and see, touch, hear and smell, the world of living things, people, consciousness, free will, meaning and value-how can all of this exist and best flourish embedded as it is in the physical universe? This problem becomes serious the moment people begin to take versions of physicalism seriously. This began to happen when modern science-or rather natural philosophy-emerged in the 17th century. Natural philosophers began to take seriously the idea that the world is made up exclusively of corpuscles, devoid of colour, sound and smell; or, a bit more generally, the universe is such that everything occurs in accordance with precise laws, the book of nature being written in the language of mathematics, as Galileo put it.

Philosophy ought to have taken this human world/physical universe problem, as we may call it, as the fundamental problem of all of thought and life. A basic task of philosophy ought to have been to keep alive awareness of the fundamental character of this problem, the need to put it at the heart of academic thought and education so that fruitful interactions between this problem and all our more

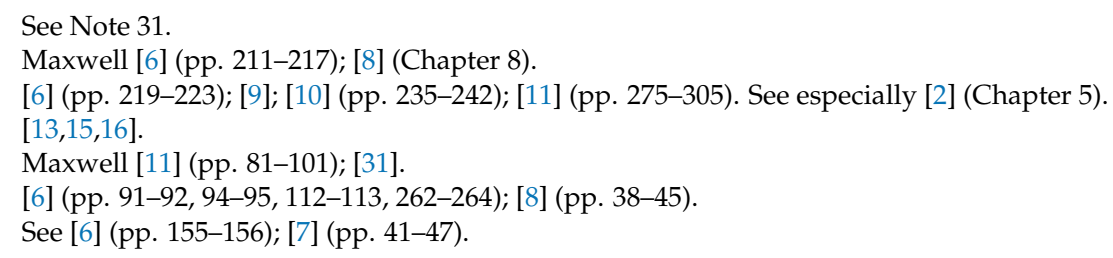


specialized, particular problems, may be explored. Philosophy ought to have kept alive rational-that is, imaginative and critical—attempts to solve the problem, or at least improve attempts to solve it.

If this had been done, long ago in the 18th century, let us say, perhaps as a fundamental idea and endeavour of the Enlightenment, we would today have a very different kind of academic enterprise, and a very different modern world, from what confronts us today. Our institutions of learning, our universities and schools, would have given intellectual priority to problems of living-problems of how what is of value in life is to be realized, at all levels from the personal, the institutional and social, to the global. Science would of course have been important, but not fundamental. "What is of value in life and how is it to be realized?" would have been the fundamental question. Scientific answers to questions about the world and ourselves as a part of the world, would have facilitated and constrained answers to the more basic questions about what is genuinely of value in life and how it is to be realized.

Why did philosophy fail to give due prominence to our fundamental problem-the human world/physical universe problem? Because philosophy became mesmerized by Descartes's attempted solution to the problem.

Descartes tried to solve the human world/physical universe problem by segregating the human and the physical into two distinct domains. On the one hand there is the physical universe; and on the other, there is mind-consciousness, the soul, all that which is of value. And having split asunder the two components of the original problem in this way, the problem then became to see how these two components could be related, or could interact. How could there be minds interacting with physical brains? Thus was born the mind-body problem of modern philosophy.

Most philosophers who came after Descartes agreed that Descartes's attempted solution is untenable. But, instead of returning to the original problem that, implicitly, gave rise to Descartes's attempted solution in the first place, paradoxically they struggled with the implications of Descartes's untenable solution. They agonized about how we can know anything about the physical universe if we are confined to the universe of mind. They struggled to understand how physical events in the brain could cause, and be caused by, mental events in the mind. They grappled with the problem of how there could be free will if determinism held sway in the physical universe. Singularly, they failed to return to the more fundamental human world/physical universe problem that Descartes may be construed to have tried, and failed, to solve. Having rejected Descartes's attempted solution, the rational thing to do would have been to return to the problem it sought to solve. Philosophy did not begin to do that. Instead it struggled to solve problems generated by Cartesian Dualism, even though Cartesian Dualism itself had been rejected!

Elsewhere, I have tried to spell out the consequences, for philosophy, for natural science, for social science, for academic inquiry as a whole, for our modern world, of returning to our fundamental problem, and placing that at the heart of education and academic thought: [2,7,10,22,32,33]. The outcome is not just natural philosophy, but a new kind of academic inquiry that gives intellectual priority to problems of living, and actively seeks to help humanity resolve conflicts and problems of living in increasingly cooperatively rational ways. The basic intellectual aim of inquiry becomes, not knowledge, but rather wisdom-wisdom being the capacity, active endeavour and desire to realize (apprehend and create) what is of value in life for oneself and others, wisdom in this sense including knowledge, technological know-how, and understanding, but much else besides.

We have failed to develop academic inquiry so that it is rationally designed and devoted to help promote human welfare by intellectual, educational and technological means. Academic inquiry as it exists at present is not best designed to help humanity learn how to resolve its conflicts and problems of living so that we may make progress towards a better, wiser world. And this failure is, at root, a philosophical failure, a failure to get clear about what the overall aims and methods of rational inquiry ought to be. Involved are two major philosophical failures: first, the failure to solve the problem of induction; and second, the failure even to formulate properly our fundamental problem—the human world/physical universe problem. 
We urgently need to bring about a revolution in humanity's institutions of learning, so that humanity may be able to begin to learn how to make social progress towards a better, wiser world.

Funding: This research received no external funding.

Conflicts of Interest: The author declares no conflict of interest.

\section{References}

1. Newton, I. Principia; University of California Press: Berkeley, CA, USA, 1962.

2. Maxwell, N. In Praise of Natural Philosophy; McGill-Queen's University Press: Montreal, QC, Canada, 2017.

3. Harman, G. The Inference to the Best Explanation. Philos. Rev. 1965, 74, 88-95. [CrossRef]

4. Hume, D. A Treatise of Human Nature; Dent, London, UK, 1959; Volume 1.

5. Howson, C. Hume's Problem; Oxford University Press: Oxford, UK, 2000.

6. Maxwell, N. The Comprehensibility of the Universe; Clarendon Press: Oxford, UK, 1998.

7. Maxwell, N. Is Science Neurotic? Imperial College Press: London, UK, 2004.

8. Maxwell, N. Understanding Scientific Progress; Paragon House: St. Paul, MN, USA, 2017.

9. Maxwell, N. The Rationality of Scientific Discovery. Philos. Sci. 1974, 41, 123-153. [CrossRef]

10. Maxwell, N. From Knowledge to Wisdom: A Revolution in the Aims and Methods of Science; Blackwell: Oxford, UK, 1984. 2nd ed., Pentire Press: London, UK, 2007.

11. Maxwell, N. Induction and Scientific Realism: Einstein versus van Fraassen. Br. J. Philos. Sci. 1993, 44, 61-79, 81-101, 275-305. [CrossRef]

12. Maxwell, N. The Need for a Revolution in the Philosophy of Science. J. Gen. Philos. Sci. 2002, 33, 381-408. [CrossRef]

13. Maxwell, N. Popper, Kuhn, Lakatos and Aim-Oriented Empiricism. Philosophia 2005, 32, 181-239. [CrossRef]

14. Maxwell, N. A Priori Conjectural Knowledge in Physics. In What Place for the A Priori? Shaffer, M., Veber, M., Eds.; Open Court: Chicago, IL, USA, 2011; pp. 211-240.

15. Maxwell, N. Popper's Paradoxical Pursuit of Natural Philosophy. In Cambridge Companion to Popper; Shearmur, J., Stokes, G., Eds.; Cambridge University Press: Cambridge, UK, 2016; pp. 170-207.

16. Maxwell, N. Unification and Revolution: A Paradigm for Paradigms. J. Gen. Philos. Sci. 2014, 45, 133-149. [CrossRef]

17. Braithwaite, R.B. Scientific Explanation; Cambridge University Press: Cambridge, UK, 1953.

18. Salmon, W.C. The Pragmatic Justification of Induction. In The Justification of Induction; Swinburne, R., Ed.; Oxford University Press: Oxford, UK, 1974; pp. 85-97.

19. Mellor, D.H. Matters of Metaphysics; Cambridge University Press: Cambridge, UK, 1991.

20. McAllister, J. Beauty and Revolution in Science; Cornell University Press: Ithaca, NY, USA, 1996.

21. Van Fraassen, B. Empiricism in the Philosophy of Science. In Images of Science; Churchland, P.M., Hooker, C.A., Eds.; University of Chicago Press: Chicago, IL, USA, 1985; pp. 245-308.

22. Maxwell, N. Karl Popper, Science and Enlightenment; UCL Press: London, UK, 2017.

23. Maxwell, N. Can there be Necessary Connections between Successive Events? Br. J. Philos. Sci. 1968, 19, 1-25. [CrossRef]

24. Maxwell, N. Towards a Micro Realistic Version of Quantum Mechanics. Parts I and II. Found. Phys. 1976, 6, 275-292. [CrossRef]

25. Maxwell, N. Quantum Propensiton Theory: A Testable Resolution of the Wave/Particle Dilemma. Br. J. Philos. Sci. 1988, 39, 1-50. [CrossRef]

26. Maxwell, N. A New Look at the Quantum Mechanical Problem of Measurement. Am. J. Phys. 1972, 40, 1431-1435. [CrossRef]

27. Maxwell, N. Instead of Particles and Fields. Found. Phys. 1982, 12, 607-631. [CrossRef]

28. Maxwell, N. Particle Creation as the Quantum Condition for Probabilistic Events to Occur. Phys. Lett. A 1994, 187, 351-355. [CrossRef]

29. Maxwell, N. Is the Quantum World Composed of Propensitons? In Probabilities, Causes and Propensities in Physics; Suárez, M., Ed.; Synthese Library, Springer: Dordrecht, The Netherlands, 2011; pp. 221-243.

30. Maxwell, N. Could Inelastic Interactions Induce Quantum Probabilistic Transitions? In Collapse of the Wave Function; Gao, S., Ed.; Cambridge University Press: Cambridge, UK, 2018; Chapter 14; pp. 257-273. 
31. Maxwell, N. Does Orthodox Quantum Theory Undermine, or Support, Scientific Realism? Philos. Q. 1993, 43, 139-157. [CrossRef]

32. Maxwell, N. Arguing for Wisdom in the University: An Intellectual Autobiography. Philosophia 2012, 40, 663-704. [CrossRef]

33. Maxwell, N. How Universities Can Help Create a Wiser World: The Urgent Need for an Academia Revolution; Imprint Academic: Exeter, UK, 2014. 